\title{
Solution Structures and Dynamic Assembly of the 24-Meric Plasmodial Pdx1-Pdx2 Complex
}

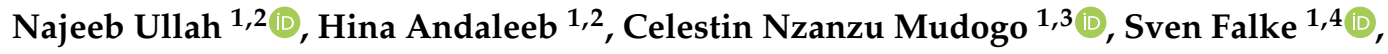 \\ Christian Betzel ${ }^{1,4, *}$ and Carsten Wrenger ${ }^{5, *}$ \\ 1 Institute of Biochemistry and Molecular Biology, Laboratory for Structural Biology of Infection and \\ Inflammation, University of Hamburg, c/o DESY, Build. 22a. Notkestr. 85, 22603 Hamburg, Germany; \\ najeeb.ullah@chemie.uni-hamburg.de (N.U.); hina.andaleeb@chemie.uni-hamburg.de (H.A.); \\ cmudogo@gmail.com (C.N.M.); falke@chemie.uni-hamburg.de (S.F.) \\ 2 Department of Biochemistry, Bahauddin Zakariya University, Multan-60800, Punjab, Pakistan \\ 3 Department of Basic Sciences, School of Medicine, University of Kinshasa, Kinshasa BP834 KinXI, Congo \\ 4 The Hamburg Centre for Ultrafast Imaging (CUI), Luruper Chaussee 149, 22761 Hamburg, Germany \\ 5 Unit for Drug Discovery, Department of Parasitology, Institute of Biomedical Sciences, \\ University of São Paulo, Av. Prof. Lineu Prestes 1374, 05508-000 São Paulo-SP, Brazil \\ * Correspondence: Christian.Betzel@uni-hamburg.de (C.B.); cwrenger@icb.usp.br (C.W.); \\ Tel.: +49-(40)-8998-4744 (C.B.); +55-(11)-3091-7265 (C.W.)
}

Received: 22 June 2020; Accepted: 17 August 2020; Published: 19 August 2020

check for updates

\begin{abstract}
Plasmodium species are protozoan parasites causing the deadly malaria disease. They have developed effective resistance mechanisms against most antimalarial medication, causing an urgent need to identify new antimalarial drug targets. Ideally, new drugs would be generated to specifically target the parasite with minimal or no toxicity to humans, requiring these drug targets to be distinctly different from the host's metabolic processes or even absent in the host. In this context, the essential presence of vitamin $\mathrm{B}_{6}$ biosynthesis enzymes in Plasmodium, the pyridoxal phosphate (PLP) biosynthesis enzyme complex, and its absence in humans is recognized as a potential drug target. To characterize the PLP enzyme complex in terms of initial drug discovery investigations, we performed structural analysis of the Plasmodium vivax PLP synthase domain (Pdx1), glutaminase domain (Pdx2), and Pdx1-Pdx2 (Pdx) complex (PLP synthase complex) by utilizing complementary bioanalytical techniques, such as dynamic light scattering (DLS), X-ray solution scattering (SAXS), and electron microscopy (EM). Our investigations revealed a dodecameric Pdx1 and a monodispersed Pdx complex. Pdx2 was identified in monomeric and in different oligomeric states in solution. Interestingly, mixing oligomeric and polydisperse Pdx 2 with dodecameric monodisperse Pdx 1 resulted in a monodispersed Pdx complex. SAXS measurements revealed the low-resolution dodecameric structure of $\mathrm{Pdx} 1$, different oligomeric structures for $\mathrm{Pdx} 2$, and a ring-shaped dodecameric Pdx1 decorated with Pdx2, forming a heteromeric 24-meric Pdx complex.
\end{abstract}

Keywords: pyridoxal phosphate (PLP) synthase; Pdx1 and Pdx2; solution structure; protein-protein interactions; reversible oligomerization; drug discovery; malaria parasites; vitamin B6 synthesis

\section{Introduction}

Malaria, with more than 250 million humans infected annually and up to 0.5 million fatalities [1], highlights an urgent need to identify and discover new antimalarial drugs [2] for use against the human pathogens Plasmodium falciparum, P. vivax, Plasmodium knowlesi, Plasmodium malariae, and Plasmodium ovale, in particular [2,3]. Drug targets that interfere with the metabolism of the parasite, like the vital vitamin pathways, are the focus of the latest drug discovery investigations [4]. Although P. falciparum is responsible for the majority of the cases and deaths, P. vivax has a wider geographical distribution 
and is the causative organism of almost half of malaria cases outside Africa. Further, it generates re-emerging quiescent liver-stage parasites, causing repeated clinical episodes of malaria due to a single infection. Therefore, the World Health Organization (WHO) currently prioritizes investigations targeting P. vivax rather than other Plasmodium species [5,6].

Pyridoxal-5-phosphate (PLP), the active cofactor for more than 100 vitamin $\mathrm{B}_{6}$-dependent enzymes, results from the transformation of its precursors, namely pyridoxine, pyridoxamine, and pyridoxal. $[7,8]$. Bacteria, fungi, and plants synthesize PLP de novo via the vitamin $B_{6}$ biosynthesis pathway, whereas mammals are entirely dependent on its uptake [9]. Biochemical analysis unveiled that the PLP synthase complex functions as a glutamine amidotransferase to produce PLP, where Pdx2 functions as the glutaminase domain to metabolize glutamine to ammonia and $\operatorname{Pdx} 1$ as the synthase domain, which utilizes the substrates ribose-5-phosphate, glyceraldehyde-3-phosphate, and ammonia [10,11]. The ammonia produced by $\mathrm{Pdx} 2$ is supplied to the active site of the synthase domain (Pdx1) through a hydrophobic ammonia tunnel [12].

Biochemical and biophysical characterizations of P. falciparum and Bacillus subtilis PLP synthases revealed that eukaryotic and prokaryotic Pdx complexes have substantial structural differences [12,13]. The crystal structures of Pdx1 from Geobacillus stearothermophilus (4WXZ), B. subtilis (2NV1), [14,15], and Mycobacterium tuberculosis (4JDY) [16] unveiled a dodecameric structure. However, for Saccharomyces cerevisiae Pdx1, analytical ultracentrifugation (AUC) and X-ray crystallography studies revealed a hexamer (3FEM) [17]. Crystal structures of Pdx2 (1R9G, 2ABW) [18,19], and the PLP synthase complex were reported for prokaryotes with protein data bank (pdb) codes 2NV2, 2ISS, 4WXY [15,20,21] and for a Plasmodium chimeric complex (4ADS) [22]. The Pdx complex is more stable in Plasmodium than in Bacillus [12], and Pdx1 and the Pdx1-Pdx2 complex are substantially different in terms of oligomerization and stability in solution [12,22].

Knowing the differences between eukaryotic and prokaryotic Pdx1 and Pdx complexes and gaining insight into plasmodial Pdx1 and Pdx complex dynamic oligomerization behaviors in solution would support drug discovery targeting the Pdx complex. Therefore, we investigated P. vivax $\mathrm{Pdx} 1$ and $\mathrm{Pd} 22$ and the $\mathrm{Pdx}$ complex by applying complementary bioanalytical techniques. The data obtained by applying dynamic light scattering (DLS), small angle X-rays scattering (SAXS), and electron microscopy (EM) provide insights into the structure, dynamics, and oligomerization behaviors of $\mathrm{Pdx} 1$ and $\mathrm{Pdx} 2$ and their interactions in solution.

\section{Results}

\subsection{Expression and Purification}

$P d x 1$ and $P d x 2$ genes were successfully overexpressed and corresponding active proteins with molecular weights of 35 and $26 \mathrm{kDa}$, respectively, were purified using affinity and size-exclusion chromatography (SEC). Using SEC, Pdx1 was eluted at $170 \mathrm{~mL}$ (Figure 1a). The elution profile for Pdx1, obtained by applying a Superdex S200 26/60 column for purification, is shown in Figure 1a (green). The protein fraction collected from corresponding peak A was evaluated by SDS-PAGE (Figure 1c,d, lane A), indicating a dodecamer in the solution. Pdx2 was eluted at 230-256 mL (Figure 1a, red), with its corresponding peaks A and B evaluated by PAGE (Figure 1c,d, lane B) and a monomer and different oligomers indicated in solution. Pdx1 and Pdx2 were mixed in a 1:1 molar ratio and applied to a Superose-6-increase column. The elution profile is shown in Figure 1b, with peak A corresponding to the $P v \mathrm{Pdx} 1 / \mathrm{Pv} \mathrm{Pdx} 2$ complex, confirmed via SDS-PAGE (Figure 1c, lane D). The Pdx1-Pdx2 complex was eluted at approximately $15 \mathrm{~mL}$, with a corresponding molecular weight of approximately $700 \mathrm{kDa}$ (Figure 1d, lane D). This chromatography fraction was assessed via SDS-PAGE, showing two bands at 35 and $26 \mathrm{kDa}$ corresponding to reduced monomeric Pdx1 and Pdx2 (Figure 1c). The purified Pdx complex was further characterized for functionality according to [7], and by native PAGE, a band corresponding to a molecular weight of 650-700 kDa (Figure 1d) was observed. 


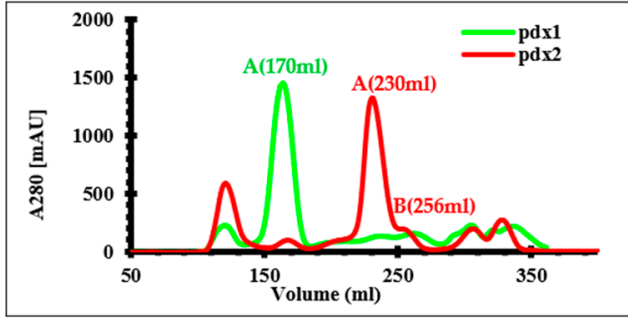

(a)

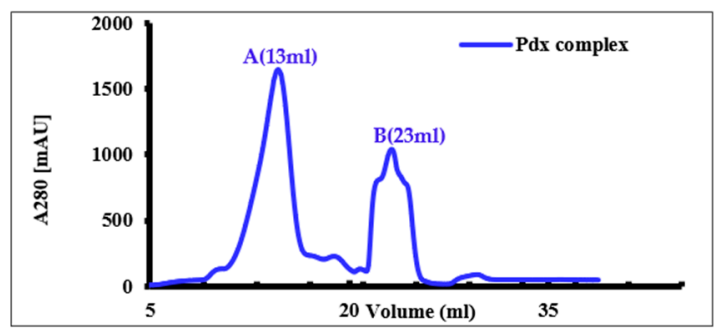

(b)

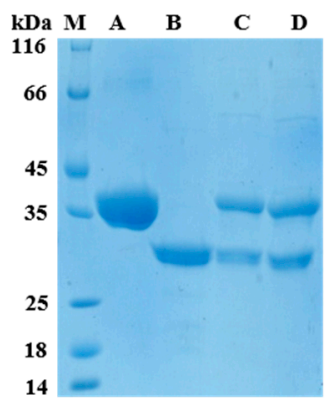

(c)

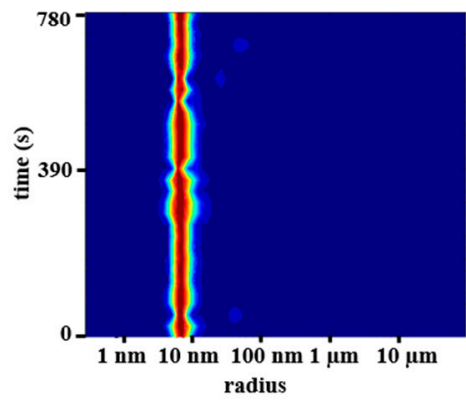

(f)

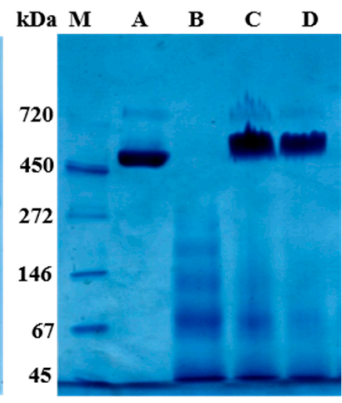

(d)

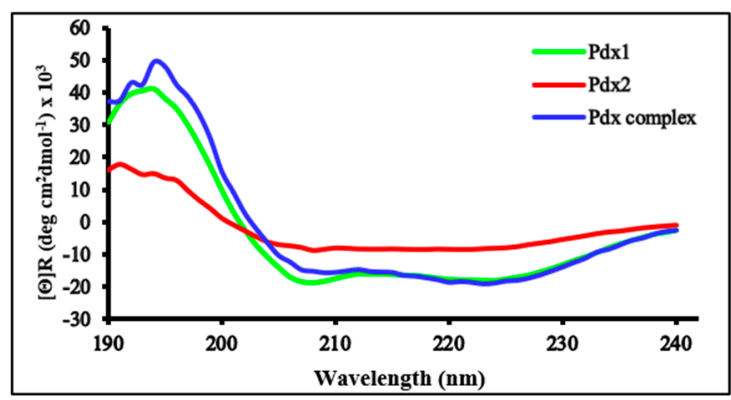

(e)

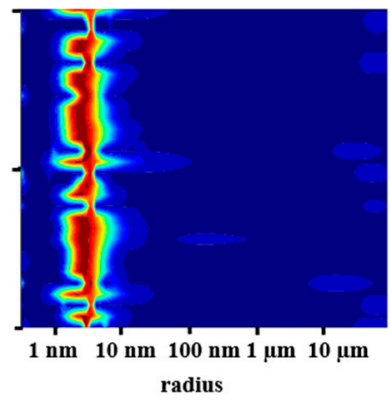

(h)

Figure 1. Size-exclusion chromatography (SEC) elution profile of (a) $\mathrm{Pdx} 1$ and $\mathrm{Pdx} 2$ using a Superdex 200 26/60 column and (b) the Pdx complex using a Superose-6-increase 10/300 column; (c) SDS-PAGE of the purified samples: lane A corresponds to Pdx1, lane B to Pdx2, lane C to the Pdx1-Pdx2 complex before SEC, and D shows the SEC-purified Pdx complex; (d) Native PAGE showing the unreduced form of the Pdx proteins: lane A: Pdx1 dodecamer; lane B: Pdx2 in different oligomeric states; lane C: a Pdx1 and Pdx2 sample mixed in 1:1 molar ratio applied to SEC; lane D: purified Pdx complex at approximately $700 \mathrm{kDa}$; (e) Circular Dichroism (CD) spectroscopy results showing secondary structure contents of Pdx1 (green), the complex (blue) with $\alpha$-helices and $\beta$-sheets as predominant secondary structure elements, and $\operatorname{Pdx} 2$ (red) with $\alpha$-helices, $\beta$-sheets, and some random coils. Experimental CD data obtained for PvPdx2 were compared with different secondary structure content predicting servers, i.e., SOMPA ( $\alpha$-helices 33, $\beta$-sheets 21 , and coils 40$)$, DSSP ( $\alpha$-helices 29 , $\beta$-sheets 32, and coils 39), and Predict Protein ( $\alpha$-helices 29, $\beta$-sheets 27, and coils 44). Calculated data are consistent with the experimental data. Dynamic light scattering (DLS) analysis of (f) dodecameric $\operatorname{Pdx} 1\left(\mathrm{R}_{\mathrm{H}}=7.3 \pm 0.9 \mathrm{~nm}\right),(\mathrm{g})$ monodispersed saturated Pdx1-Pdx2 complex $\left(\mathrm{R}_{\mathrm{H}}=9.7 \pm 0.2 \mathrm{~nm}\right)$, and (h) monomeric $\operatorname{Pdx} 2\left(\mathrm{R}_{\mathrm{H}}=2.8 \pm 0.4 \mathrm{~nm}\right)$.

\subsection{Circular Dichroism (CD) Spectroscopy and DLS Investigations}

Secondary structure information obtained by applying CD spectroscopy indicated $52 \% \alpha$-helix and $20 \% \beta$-sheet contents for Pdx1,36\% and 34\% for Pdx2, respectively, and $54 \%$ and $25 \%$ for the complex, respectively, with corresponding mean RMS (root mean square) values of 8.4,6.2, and 6.5, respectively, using the JASCO Spectra Manager software suite for data evaluation (Figure 1e) [23]. The CD data and corresponding secondary structure contents were compared with the secondary structure content 
of the homologous PfPdx2 crystal structure (pdb code: 2ABW) [19]. Further, the PvPdx2 secondary structure content was calculated by applying different secondary structure prediction servers, i.e., SOPMA (self-optimized prediction method with alignment), DSSP (define secondary structure of proteins), and Predict Protein. The resulting values are described in the legend of Figure 1e.

Dynamic light scattering is a useful technique to investigate the size distribution and dispersity of nanoparticles or macromolecular complexes in solution [24,25]. For the monodispersed P. vivax $\mathrm{Pdx} 1$ solution, DLS showed a hydrodynamic radius of $\mathrm{R}_{\mathrm{H}}=7.3 \pm 0.9 \mathrm{~nm}$ (Figure 1f), confirming its dodecameric state in solution [22]. For the Pdx complex, a hydrodynamic radius of $9.7 \pm 0.2 \mathrm{~nm}$ was observed (Figure 1g). For Pdx2, DLS experiments revealed a concentration and time-dependent oligomerization tendency, with resulting $\mathrm{R}_{\mathrm{H}}$ values of $2.8 \pm 0.4 \mathrm{~nm}$ at $1.0 \mathrm{mg} \mathrm{mL}^{-1}$ concentration, corresponding to approximately $35 \mathrm{kDa}$ (Figure $1 \mathrm{~h}$ ) and $3.5 \pm 0.4 \mathrm{~nm}$ at $1.6 \mathrm{mg} \mathrm{mL}^{-1}$ and $4.2 \pm 0.4 \mathrm{~nm}$ at $2.5 \mathrm{mg}$, corresponding to approximately $60 \mathrm{kDa}$ and $90 \mathrm{kDa}$, respectively (Supplementary Figure $\mathrm{S} 1 \mathrm{a}, \mathrm{b})$. Most interestingly, mixing an oligomeric Pdx2 suspension, which was shown via DLS to have a multiple radius distribution (Supplementary Figure S1a-d) with dodecameric monodispersed Pdx1, resulted in a monodispersed Pdx complex with a hydrodynamic radius of $10.9 \pm 1.4 \mathrm{~nm}$ (Supplementary Figure S1e).

\subsection{Reversible Oligomerization of $P d x 2$ by DLS Investigation}

We analyzed the reversible oligomerization tendency of Pdx2 by applying time-resolved DLS and mixing nonhomogeneous Pdx2 oligomers with homogenous dodecameric Pdx1. A Pdx complex formation was observed by utilizing dodecameric $\mathrm{Pdx} 1$ and $\mathrm{Pdx} 2$ (monomeric and oligomeric) in independent experiments. The plots in the center display the respective size distribution over time. The abundance of radii (number of particles scattering the laser light) is color-coded from blue (low) to red (high).

After mixing dodecameric $\mathrm{Pdx} 1$ and monomeric $\mathrm{Pdx} 2$ in a 1:1 molar ratio, interactions between the two proteins were gradually established, resulting in a monodispersed Pdx complex at a concentration of $5 \mathrm{mg} \mathrm{mL}^{-1}$ with $\mathrm{R}_{\mathrm{H}}=10.4 \mathrm{~nm}$ (Figure 2a and Supplementary Video S1). By mixing a nonhomogeneous solution of oligomeric Pdx2 with homogenous dodecameric Pdx1, we observed the formation of a $\mathrm{Pdx}$ complex with slightly higher $\mathrm{R}_{\mathrm{H}}$ value distribution over time. The obtained suspension did not entirely show a monodispersed Pdx complex; presumably some high-molecular-weight Pdx2 multimers remained in solution $12 \mathrm{~h}$ after measurement (Figure $2 \mathrm{~b}$ and Supplementary Video S2). In addition, a monodispersed $\mathrm{Pdx}$ complex solution was obtained after centrifuging suspensions of homogenous Pdx1 and nonhomogeneous oligomeric $\mathrm{Pdx} 2$ at $10,000 \times g$ for $10-15 \mathrm{~min}$, as shown in Supplementary Figure S1e. 

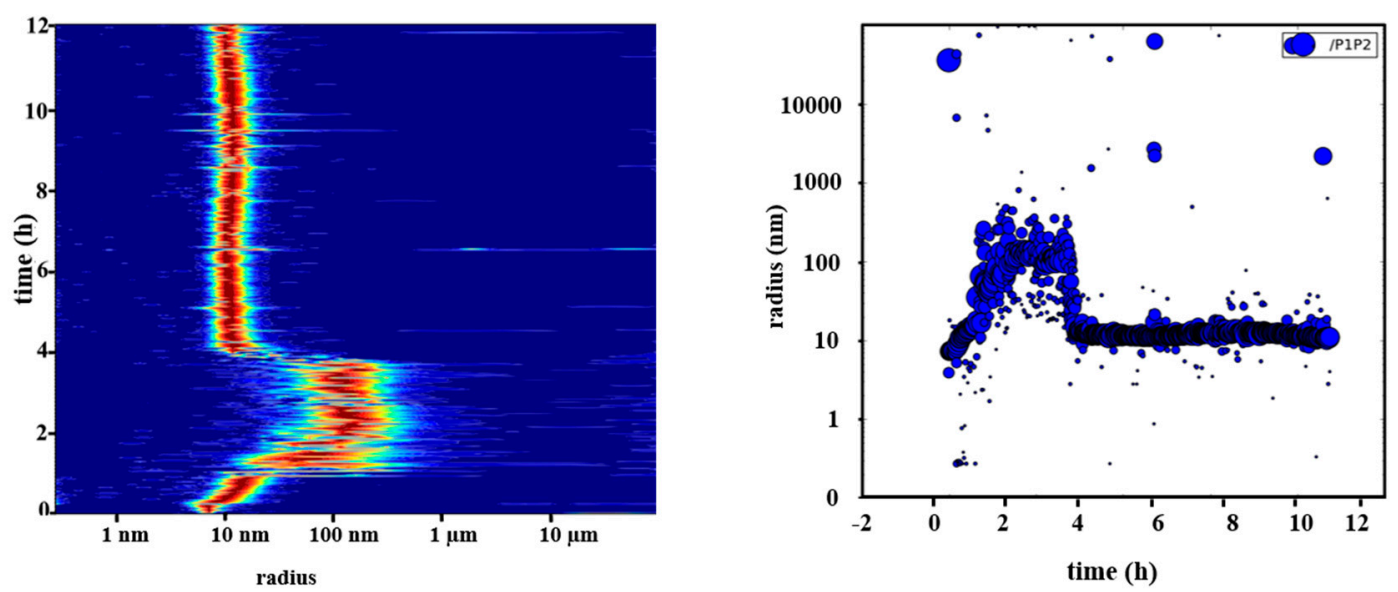

(a)
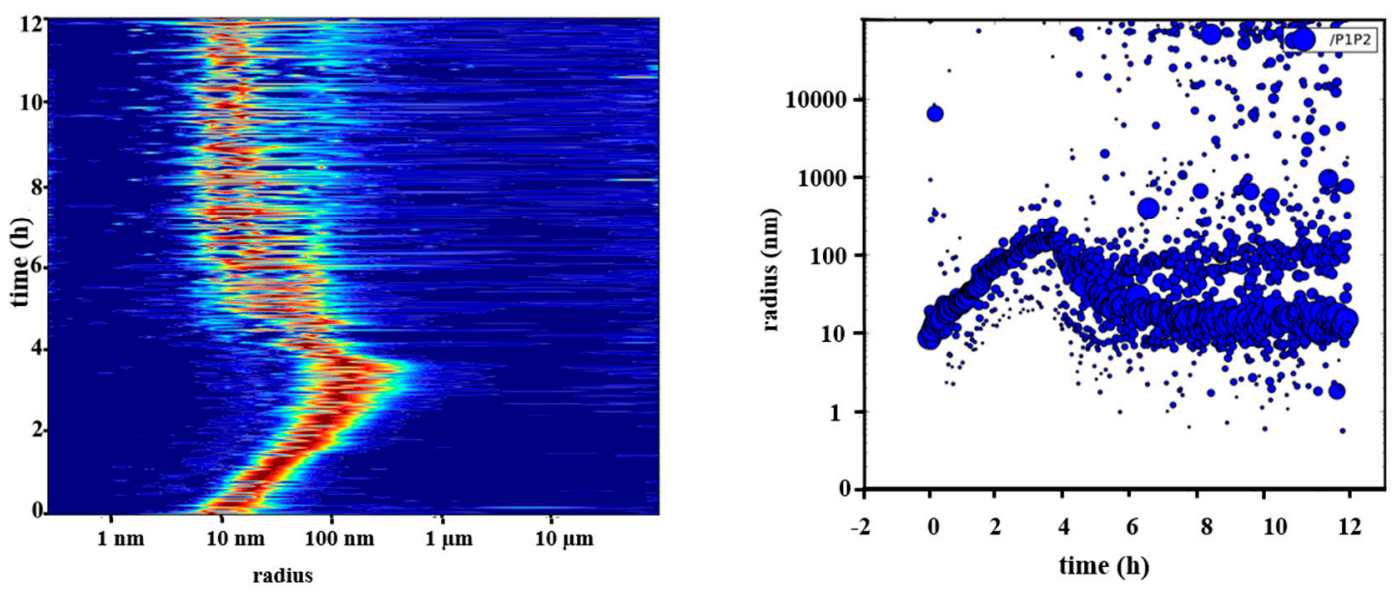

(b)

Figure 2. Figures on the left in red indicate the particle size distribution, with the y-axis corresponding to the time course of the experiment and the horizontal axis showing the radius distribution. For figures on the right side, the axes are inverse. (a) Particle size distribution of dodecameric Pdx1 mixed with monomeric Pdx2 measured over $12 \mathrm{~h}$ after applying DLS. First, dodecameric Pdx1 was measured for 25 min after a solution of $\operatorname{Pdx} 2$ was added. After mixing $\operatorname{Pdx} 1$ and $\mathrm{Pdx} 2$, the predominant particle radii distribution shifted toward larger radii of approximately 100-300 $\mathrm{nm}$. The high-molecular-weight particles $\left(R_{H}>100 \mathrm{~nm}\right)$ remained for two to three hours. Subsequently, the particle radii shifted to approximately 10-14 nm, corresponding to the radius of the Pdx complex; (b) DLS measurements of a suspension containing dodecameric $\mathrm{Pdx} 1$ and oligomeric $\mathrm{Pdx} 2$ recorded over time. After 15 initial measurements of dodecameric Pdx1, oligomeric Pdx2 was added and the radius profile shifted toward higher radius values, i.e., oligomeric Pdx2 interacted with Pdx1 to form the Pdx complex. After 4-5 h, the equilibrium shifted toward a radius corresponding to the Pdx complex radius with some remaining polydispersity. As seen from the radius profile, some large oligomeric particles, presumably corresponding to Pdx2 oligomers, remained in solution.

\subsection{SAXS Analysis}

To further analyze the molecular structure, dynamicity, and oligomerization of the Pdx complex and its subunits in solution, monodispersed solutions of Pdx1, Pdx2, and the Pdx complex were subjected to SAXS experiments; their respective averaged scattering intensity profiles are displayed in Figure 3a-c. Guinier analysis using the Guinier approximation and radius of gyration $\left(R_{G}\right)$ as determined by AUTOGNOM provided $R_{G}$ values for $\operatorname{Pdx1}$, Pdx2, and their complex, which are summarized in Table 1. 


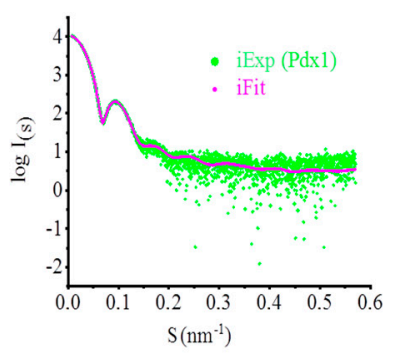

(a)

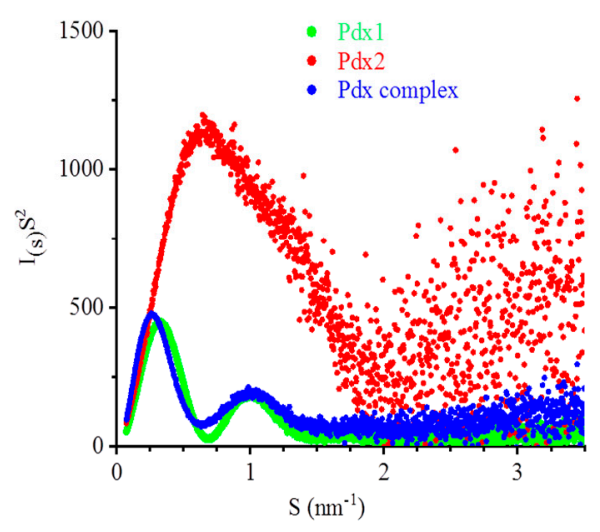

(d)

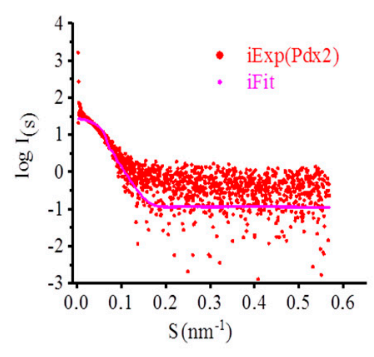

(b)

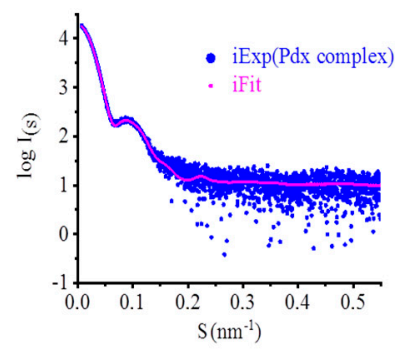

(c)

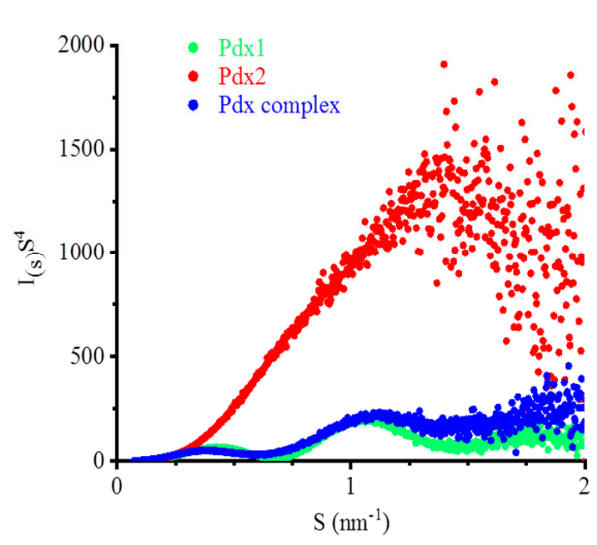

(e)

Figure 3. Processed solution scattering intensity pattern of (a) Pdx1 (green), (b) Pdx2 (red), and (c) the Pdx complex (blue) in arbitrary intensity units. Their fits with selected homolog structures are shown in magenta (Pdx1: PDB ID 4ADU; Pdx2: PDB ID 2ABW; Pdx complex: PDB ID 4ADS). Data were processed and evaluated by applying the programs CRYSOL and SASREF; (d) dimensionless Kratky plots indicate globular and compact structures for Pdx1 and the Pdx complex and a more flexible structure for $\mathrm{Pdx} 2$ ( $\mathrm{Pdx} 1$ is shown in green, $\mathrm{Pdx} 2$ in red, and the Pdx1-Pdx2 complex in blue); (e) Porod-Debye plots of the X-ray solution scattering (SAXS) data including the color-coding used in (d).

The distance distribution function $P(r)$ indicated a maximum diameter $\left(D_{\max }\right)$ of $15 \mathrm{~nm}$ and a nearly globular shape for $\mathrm{Pdx} 1$, a $D_{\max }$ of $11.3 \mathrm{~nm}$, and a rod-shaped structure for Pdx2, as well as $D_{\max }$ of $22.2 \mathrm{~nm}$ for the Pdx complex with a more spherical but slightly extended structure (Supplementary Figure $\mathrm{S} 2$ and Table 1). Kratky plots $\left(\mathrm{I}_{(\mathrm{S})} \mathrm{S}^{2}\right.$ versus $\mathrm{S}$ ) obtained from the scattering data were used to verify the flexibility of the proteins. Based on the Kratky plots of the scattering data, Pdx1 and the $\mathrm{Pdx}$ complex represented rigid and compactly folded particles (Figure 3d). In contrast, the Kratky plot of Pdx2 indicated a significant intrinsic flexibility [26] (Figure 3d). Porod-Debye plots $\left(\mathrm{I}_{(\mathrm{S})} \mathrm{S}^{4}\right.$ versus $S$ ) of the scattering data of the three proteins showed plateaus, indicating that Pdx1 and the Pdx complex lack disordered regions, whereas $\mathrm{Pdx} 2$ is more flexible in solution (Figure 3e). The shape factor $\left(\rho=R_{G} / R_{H}\right)$ [27] gave a value of 0.69 for Pdx1 and 0.71 for the Pdx complex, indicating both as nearly spherical globular particles, whereas $\mathrm{Pdx} 2$ exhibited values ranging from 0.931 to 1.18 , indicative of a flexible ellipsoidal structure [28]. All experimental parameters are summarized in Table 1. 
Table 1. SAXS data collection and analysis parameters.

\begin{tabular}{|c|c|c|c|}
\hline & Pdx1 & $\operatorname{Pdx} 2$ & Pdx Complex \\
\hline Data collection parameters & & \\
\hline X-ray source & \multicolumn{3}{|c|}{ PETRA III; EMBL beamline P12 } \\
\hline Wavelength (nm) & \multicolumn{3}{|c|}{0.124} \\
\hline Detector distance (m) & \multicolumn{3}{|c|}{3.1} \\
\hline Temperature (K) & 293 & 283 & 293 \\
\hline \multicolumn{4}{|l|}{ Structural parameters } \\
\hline$I(0)(P(r)$ function $)$ & $10,720 \pm 5.93$ & $9161 \pm 41.47$ & $19380 \pm 23.04$ \\
\hline$I(0)$ (Guinier/Auto $\left.R_{\mathrm{G}}\right)$ & $10,727.20 \pm 11.61$ & $9307.96 \pm 101.56$ & $19,658.30 \pm 28.99$ \\
\hline$I(0)(\mathrm{SAXSMoW})$ & $10,751.50$ & 33.64 & $19,592.77$ \\
\hline $\mathrm{R}_{\mathrm{G}}(\mathrm{nm})(P(r)$ function $)$ & $5.00 \pm 0.01$ & $3.26 \pm 0.02$ & $6.83 \pm 0.01$ \\
\hline $\mathrm{R}_{\mathrm{G}}(\mathrm{nm})\left(\right.$ Guinier/AutoR $\left.\mathrm{R}_{\mathrm{G}}\right)$ & $5.05 \pm 0.06$ & $3.24 \pm 1.36$ & $6.95 \pm 0.11$ \\
\hline $\mathrm{R}_{\mathrm{G}}(\mathrm{nm})($ based on SAXSMoW) & 5.07 & 3.52 & 6.89 \\
\hline $\mathrm{qR}_{\mathrm{G}}$ limit (from Guinier/AutoR $\mathrm{R}_{\mathrm{G}}$ ) & 1.12 & 1.29 & 1.15 \\
\hline $\mathrm{qR}_{\mathrm{G}}$ limit (from SAXSMoW) & 1.29 & 1.30 & 1.29 \\
\hline$D_{\max }(\mathrm{nm})$ (from $P(r)$ function) & 15.0 & 11.3 & 22.2 \\
\hline Porod volume estimate $(\mathrm{nm})$ & 609.54 & 75.88 & 1733.18 \\
\hline $\mathrm{P}=\mathrm{R}_{\mathrm{G}} / \mathrm{R}_{\mathrm{H}}$ & 0.69 & $0.93-1.18$ & 0.71 \\
\hline \multicolumn{4}{|l|}{ Molecular mass determination $(\mathrm{kDa})$} \\
\hline Molecular mass (SAXSMoW) & 418.2 & 67.0 & 735.8 \\
\hline Molecular mass (amino acids sequence) & 417.3 & 26.1 & 730.3 \\
\hline Oligomeric state & Dodecamer & Oligomer & 24-mer \\
\hline
\end{tabular}

The obtained scattering amplitudes for Pdx proteins were processed by using the software PRIMUS [29] and were compared to homologous crystal structures of P. falciparum and P. berghei with the following pdb codes using CRYSOL: Pdx1, 4ADU; Pdx complex, 4ADS, and Pdx2, 2ABW [30] (Figure $3 \mathrm{a}-\mathrm{c}$ ). The experimental scattering curves, when compared with the calculated scattering amplitudes, confirmed structural similarities to the respective homologous three-dimensional structures over a wide range of angles. 3D models for $\mathrm{Pdx} 1, \mathrm{Pdx} 2$, and the $\mathrm{Pdx}$ complex were constructed using a homology modeling approach [31,32], where Pdx1 was modeled as a dodecamer, Pdx2 in monomeric form, and the Pdx complex as 24-mer. A 3D model for Pdx1 was calculated based on PbPdx1 (pdb code: 4ADU) [22] for the Pdx complex based on the chimeric complex (4ADS) [22] and for $\mathrm{Pdx} 2$ based on the pdb entry 2ABW [19]. The reported crystal structures of $\mathrm{Pdx}$ proteins from P. falciparum and P. berghei showed sequence identities of $88 \%$ and $85 \%$ for Pdx1, respectively, and $75 \%$ and $69 \%$ for $\mathrm{Pdx} 2$, respectively, compared to the P. vivax counterparts [33]. The calculated 3D models of $\mathrm{Pdx} 1, \mathrm{Pdx} 2$, and the $\mathrm{Pdx}$ complex were superimposed by applying the program Pymol [34] with the ab-initio-built GASBOR models individually and fitted well with dummy sphere models. Pymol superimpositions were performed for Pdx1 (dodecamer) with its ab initio built model, and its fit indicated Pdx1 to be a dodecamer. Further, the SAXSMoW data resulted in a molecular mass of $418.2 \mathrm{kDa}$ for Pdx1 (theoretical mass value is $417.3 \mathrm{kDa}$ ) [35]. For the Pdx complex, a molecular mass estimation of the SAXS data indicated $735.8 \mathrm{kDa}$, which was very close to the theoretically calculated mass of $730.5 \mathrm{kDa}$. The obtained rigid body models were separately superimposed with the ab initio models of Pdx1 (Figure 4a), Pdx2 (Figure 4b), and the Pdx complex (Figure 4c), indicating an appropriate fit for $\mathrm{Pdx} 1$ and the $\mathrm{Pdx}$ complex. Due to reversible oligomerization behavior and the variable oligomeric states of $\mathrm{Pdx} 2$, rigid body models were built and calculated in regard to monomeric, dimeric, and elongated trimeric forms. The dimeric rigid body model fit best when superimposed with the ab initio models (Figure $4 \mathrm{~b}$ ), considering an additional significant contribution of trimeric $\mathrm{Pdx} 2$ to the scattering intensity pattern. A significant contribution of the trimer was also indicated by SAXSMoW data, with a corresponding molecular mass value of approximately $67 \mathrm{kDa}$ using the Pdx2 scattering profile, whereas the theoretical calculated mass of a Pdx2 monomer was $26.1 \mathrm{kDa}$. All ab initio and rigid model superpositions were performed by applying Pymol [34]. 


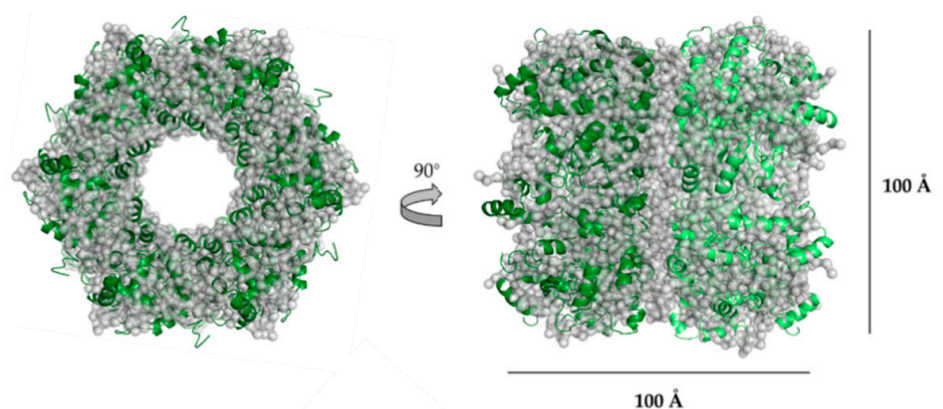

(a)

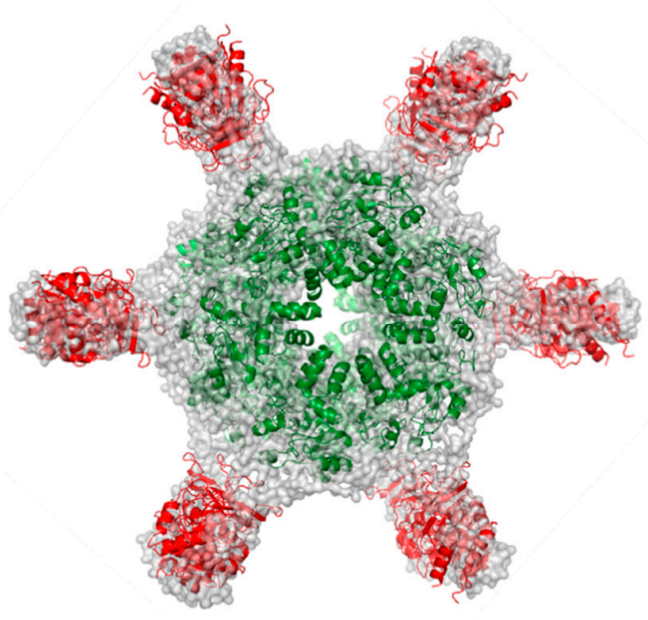

(c)

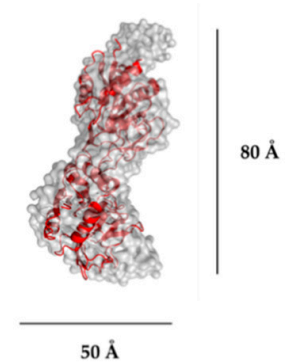

(b)

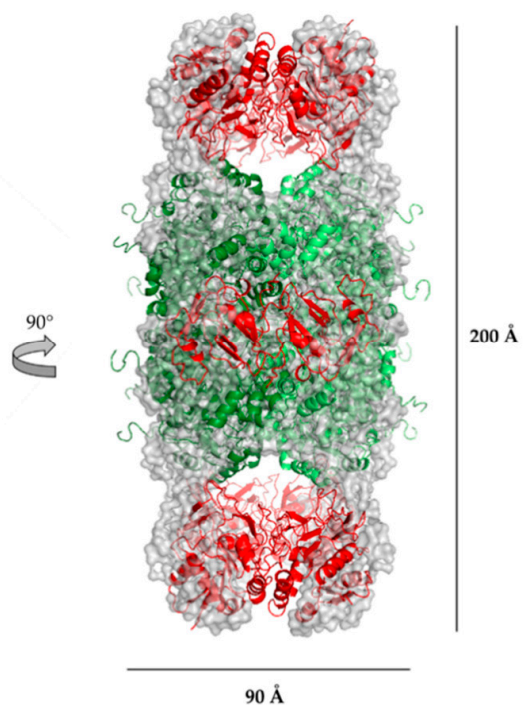

Figure 4. Ab initio GASBOR models composed of light grey-colored, chain-like dummy surface models superimposed with the rigid body SASREF models shown in cartoon representation. (a) Dodecameric Pdx1 rigid body model, i.e., one hexamer in green and one in lime color, considering a P6 symmetry superimposed with its ab initio model; (b) P1 symmetry for dimeric Pdx2 in red fitted to its ab initio model; (c) ab initio model of the Pdx1-Pdx2 (12:12) complex possessing P6 symmetry with two subunits: two hexameric ring-shaped inner cores consisting of Pdx1 (green and lime color) and a total of $12 \mathrm{Pdx} 2$ molecules (red) displaying a hexameric symmetry around the outside surface of both stacked Pdx1 hexamers corresponding to the obtained ab initio model. The ab initio model is superimposed well with the rigid body $\mathrm{Pdx}$ complex.

\subsection{Electron Microscopy (EM) Analysis}

The dodecameric P. vivax Pdx1 structure was further analyzed by negative-stain EM analysis, and the resulting images are shown in Figure $5 a, b$, thereby validating the characteristic Pdx1 dodecameric assembly made up of two hexameric rings.

The Pdx complex was also analyzed by electron microscopy, showing a partial saturation of the ring shaped Pdx1 dodecamer with Pdx2 domains (Figure 5c,d) and confirming the reported data that the attachment of $\mathrm{Pdx} 2$ to the dodecameric core of Pdx1 takes place gradually [22]. Analysis of electron microscopy figures showed that $\mathrm{Pdx} 1$ and $\mathrm{Pdx} 2$ assemble together transiently, and on average, a few Pdx2 subunits occupy Pdx1 dodecamers without preference for a distinct association pattern. Further, the reversible oligomerization behavior of Pdx2 was analyzed by EM to confirm the observed hydrodynamic radius shift recorded by DLS after mixing dodecameric Pdx1 with monomeric in one experiment and oligomeric Pdx2 in another experiment. The obtained EM micrographs confirmed the DLS data and showed that Pdx1 was complexed with Pdx2. As seen in Figure $5 d$, monomeric Pdx2 bound to dodecameric Pdx1; however, not all available vacancies were filled, whereas the binding of 
oligomeric Pdx2 to the structured Pdx1 12-mer resulted in a slightly asymmetric ring morphology of the Pdx complex particles, as shown in Figure 5e,f. The Pdx complex particles shown in Figure $5 \mathrm{f}$ were also slightly larger than those shown in Figure $5 \mathrm{~d}$.

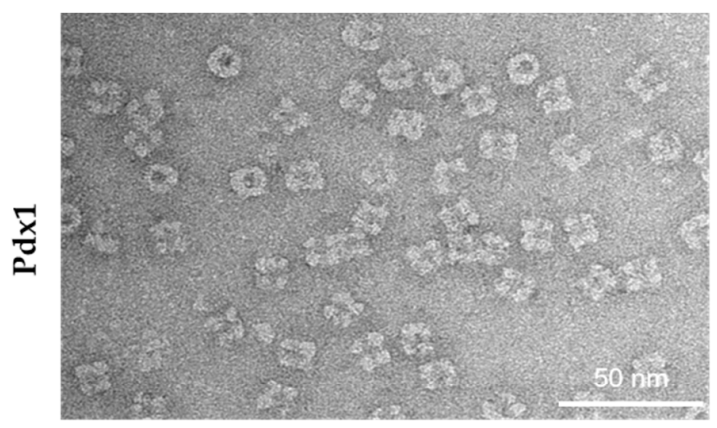

(a)

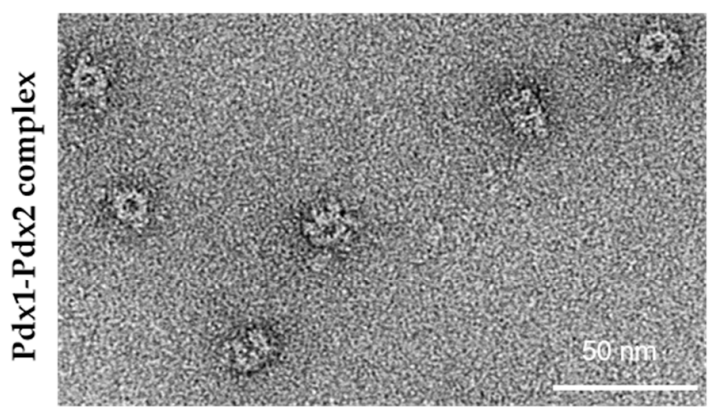

(c)

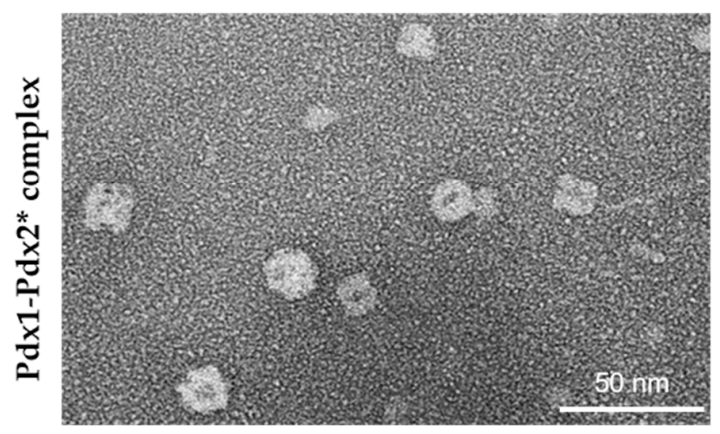

(e)
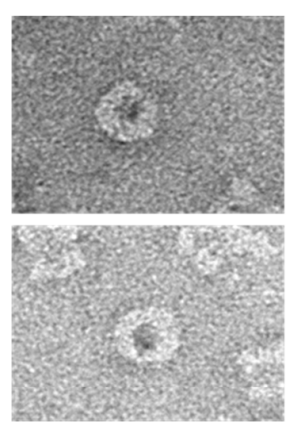

(b)
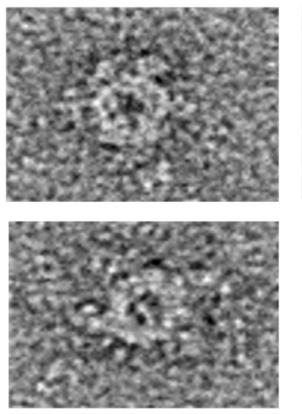

(d)

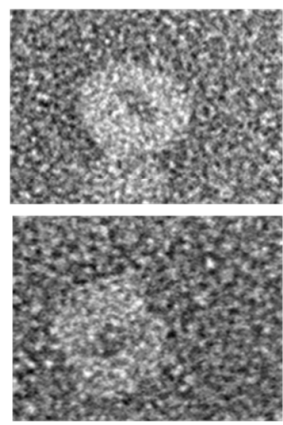

(f)
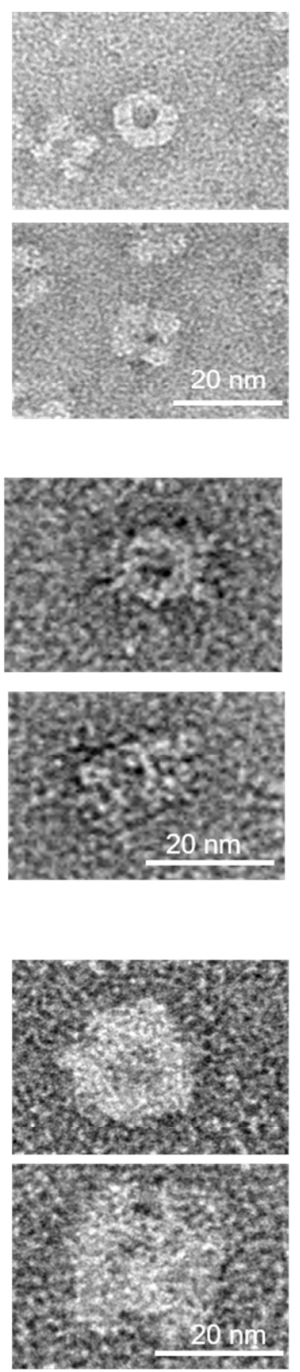

\section{* oligomeric}

Figure 5. Transmission electron micrographs of negatively stained P. vivax pyridoxal phosphate (PLP) synthase proteins. Images on the right in (b,d,f), alongside a $20 \mathrm{~nm}$ scale bar, show a zoom-in of a representative class of averaged sample pictures shown on the left in $(\mathbf{a}, \mathbf{c}, \mathbf{e})$. (a,b) Dodecameric $P d x 1$, top view, and side view with random orientations of the particles; $(\mathbf{c}, \mathbf{d})$ dodecameric Pdx1 in complex with Pdx2; for complex formation, a monomeric Pdx2 solution was provided. Pdx complex particles generally only with partially bound Pdx2 were observed and saturated (12:12) Pdx complex was rarely observed; (e,f) dodecameric Pdx1 in complex with Pdx2 oligomers. The Pdx complex was predominantly saturated with $\mathrm{Pdx} 2$, showing a slightly larger dimension than those observed for the Pdx complexes shown in (c,d), demonstrating the larger hydrodynamic radius of the corresponding Pdx complex observed by DLS (Figure 2b). * oligomeric Pdx2.

\section{Discussion}

Despite some coordinated efforts by the WHO and national health organizations, malaria still exists as an endemic disease in Africa, South America, and Asia [2]. Drug resistance for P. falciparum, P. vivax, 
and P. malariae is recognized mainly as a result of long-term overuse of antimalarial antibiotics [36]. This necessitates the identification of new antimalarial drug targets.

Plasmodium species possess the plasmodial PLP synthase, acting as a functional vitamin B6 biosynthesis pathway [9]. The class I GATase (glutamine amidotransferase) pathway consists of the synthase subunit (Pdx1) and the glutaminase subunit ( $\mathrm{Pdx} 2)$. Pdx2 produces ammonia from glutamine, which passes through a hydrophobic structural region named the ammonia tunnel and reaches the active site of $\mathrm{Pdx} 1$, where ammonia in the presence of intermediates from pentose and triose forms PLP (vitamin B6) [19] with their dissociation constant (KD) values reported from plasmodial Pdx proteins [12]. Interestingly, $\mathrm{Pdx} 1$ and $\mathrm{Pdx} 2$ require each other for activation and substrate utilization, highlighting the ammonia tunnel entrance as an interesting target region for drug design and drug discovery investigations due to its presence within the protein-protein $(\mathrm{Pd} x 1-\mathrm{Pd} \times 2)$ interface.

Pdx1 and Pdx2 are expressed throughout the erythrocytic cycle of Plasmodium, and both are cytosolic proteins able to interact and form the structural distinct $\mathrm{Pdx}$ complex, thereby generating vitamin B6 during all developmental stages of the parasite. The absence of this metabolic pathway in humans makes this vitamin B6 biosynthesis pathway an interesting target for novel antimalarial drugs [37]. In this context, it is important to understand the structure and dynamics of Pdx complex assembly, as well as to analyze the structures of the individual components, Pdx1 and Pdx2, which assemble to form the 12:12 Pdx complex. It was reported previously that B. subtilis $\mathrm{Pdx} 1$ (BsPdx1) exists in an equilibrium of hexamers and dodecamers [15]. For G. stearothermophilus, Pdx1 analytical ultra-centrifugation (AUC) studies showed a hexamer-dodecamer in solution, whereas dodecamer was present in the crystalline form [14]. Further, the eukaryote S. cerevisiae Pdx1 (ScPdx1) was reported to be hexameric in solution [17] and the eukaryotic $P f \operatorname{Pdx} 1$ was reported to exist in a dodecameric form in solution [22]. In contrast to bacterial homologs and the eukaryote S. cerevisiae, PfPdx1 even forms higher-order oligomers/fibers [22].

In terms of drug discovery investigations to treat malarial infection, we investigated $P$. vivax $\mathrm{Pdx}$ proteins by applying complementary biophysical techniques and compared our results to selected eukaryotic Pdx proteins (sequence comparison in Figure 6a,b). P. vivax Pdx1 (PvPdx1) was investigated by SEC and DLS studies, showing a dodecameric state which is stable for at least 20 days in solution (Figure 1a,f). SAXS data (Figure 4a) and EM investigations (Figure 5a,b) confirmed the dodecameric state of Pdx1 in solution and revealed a low-resolution structure. The sequence alignment (Figure 6a), of residues I-166 to L-211 showed that helices $\alpha 6, \alpha 6^{\prime}$, and $\alpha 6^{\prime \prime}$, which stabilize the dodecamer, are highly conserved among the plasmodial species and significant variations are only observed at the $\mathrm{N}$ - and C-terminus [38]. It can be assumed that the nonconserved and probably flexible C-terminus of $\mathrm{Pdx1}$, which is absent in other plasmodial homologs except for P. falciparum, is prone to degradation [22], and presumably also involved in the fiber formation observed in PfPdx1.

Higher-order oligomers and fiber formation were also reported for the $P f \mathrm{Pdx}$ complex upon storage at $4{ }^{\circ} \mathrm{C}$, as observed by analytical SEC and EM analysis [22]. For the P. vivax Pdx complex, we showed by applying SEC, DLS, SAXS, and EM that the complex was stable in solution without observing higher-order oligomers over time. Further, no significant concentration-dependent oligomerization for $\mathrm{Pdx} 1$ and the Pdx complex at concentrations ranging from 1 to $20 \mathrm{mg} \mathrm{mL}^{-1}$ was identified.

PfPdx2 was previously reported to be monomeric in solution and dimeric in the crystal lattice [19]. In contrast, we found that $\mathrm{Pv} \mathrm{Pdx} 2$ was predominantly monomeric in solution, however a fraction of oligomers were identified in solution by DLS and SAXS. Our CD results indicated approximately $64 \%$ $\beta$ sheets and coils, thereby showing that $\mathrm{Pdx} 2$ is prone to some oligomerization as increased $\beta$ sheet content increases the potential for oligomerization [39]. TANGO, a statistical mechanics algorithm based on the physicochemical principles of $\beta$-sheet formation to detect protein oligomerization, suggested that the regions 4-10, 30-35, 84-94, and 182-186 of PvPdx2, which form $\beta$ sheets, support oligomerization [40].

We further report for the first time that the oligomerization of $\mathrm{Pdx} 2$ is concentration-dependent and reversible upon interacting with dodecameric Pdx1 (Figure 2b and Supplementary Figure S1). 
The reversible oligomerization of Pdx2 toward complex formation with Pdx1 was analyzed by time-resolved DLS and confirmed by EM. EM analysis showed the Pdx complex formation after oligomeric Pdx2 interacted with dodecameric Pdx1.

For both plasmodial and bacterial PLP synthases, Pdx2 is active only in the presence of Pdx1 [19,41]. The interaction between the two proteins ( $\mathrm{Pdx} 1$ and $\mathrm{Pdx} 2$ ) is mediated by the conserved helix $\alpha \mathrm{N}$ of Pdx1 (Figure 6a) [12]. This helix is slightly longer in P. vivax than observed for other plasmodial homologs, which presumably results in different affinity between Pdx1 and Pdx2 [7] and also different stability of dodecameric Pdx1 and the Pdx complex.

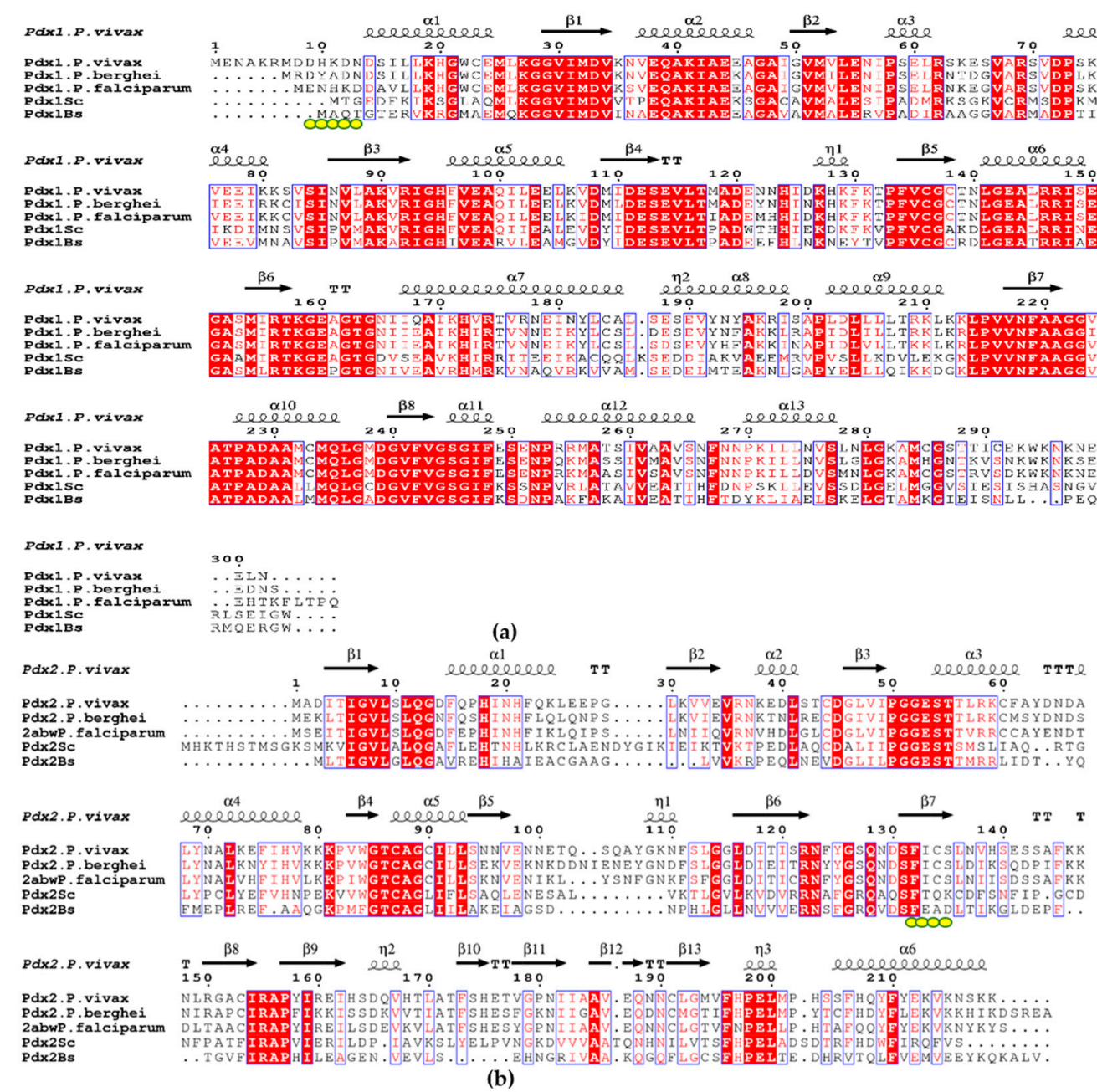

Figure 6. (a) Alignment of Pdx1 amino acid sequences from P. vivax (A5K247) P. berghei (Q4Z0E8), P. falciparum (C6KT50), S. cerevisiae (Q03148), and B. subtilis (P37527); (b) Alignment of Pdx2 amino acid sequences from P. vivax (A0A1G4GXI4) P. berghei (Q4PJX5), P. falciparum (Q8IIK4), S. cerevisiae (Q03144), and B. subtilis (P37528). The active site residue of Pdx2 H-196 is mutated in PfPdx2 to N. The red boxes with white lettering show strict identity, red letters show similarity in the region between amino acid groups, and black letters show that regions are not conserved, with TT being a strict $\beta$ turn and TTT a strict $\alpha$ turn. The Pdx1-Pdx2 contact surface involves many backbone interactions that are very much conserved in the three-dimensional structure but are not strictly conserved in the primary structure of Pdx2. Pivotal in the complex formation is the nonconserved region between $\beta-5$ and $\beta-6(\eta 1)$ and the sequence region between $\beta 6$ and $\beta 7$ of the glutaminase. The yellow dots in Pdx1 mark the $\beta N$ region, which interacts with the region (131-134) in the $\mathrm{Pdx} 2$ involved in $\beta$-completion and complex stabilization in bacterial complexes. This $\beta$-completion is not reported in plasmodial species [22], but the elongated N-terminus in PvPdx1 (which is absent in other plasmodial homologs) may have a role in complex stabilization. The sequence alignment was prepared by Espript3 [42]. 
After analyzing the PLP synthase proteins and the complex formation by applying different biophysical techniques, it can be concluded that, in comparison to other plasmodial homologs, Pdx1 and the Pdx complex from P. vivax are more stable in solution than their homologs. However, the Pdx complex can show variations in terms of attachment of monomeric and oligomeric Pdx2.

\section{Materials and Methods}

\subsection{Expression and Purification of P. vivax Pdx1, Pdx2, and Complex Formation}

Genes of Pdx1 and Pdx2 with restriction sites recognized by NdeI and XhoI were cloned into the expression vector pET31b(+) (BioCat GmbH, Heidelberg, Germany) separately. Cells of Escherichia coli strain BL21-CodonPlus-(DE3)-RIL were transformed with the expression vector, grown at $37^{\circ} \mathrm{C}$, and induced by $0.5 \mathrm{mM}$ IPTG. The filtered supernatant from cells lysed by sonication was applied to a two-step purification protocol, i.e., Ni-NTA affinity chromatography and SEC. Pure Pdx1 and Pdx2 were obtained using a Superdex 200 column (26/60, GE Healthcare, Uppsala, Sweden) and mixed in a 1:1 molar ratio and incubated overnight with $10 \mathrm{mM}$ L-glutamine. The resulting protein complex was finally purified by a Superose-6-increase column (10/300, GE Healthcare, Uppsala, Sweden).

\subsection{Dynamic Light Scattering (DLS) and Circular Dichroism (CD) Investigations}

For DLS analysis, sample solutions were measured in a quartz cuvette using the Spectroscatter-301 (Xtal Concept, Hamburg, Germany) applying a laser wavelength of $660 \mathrm{~nm}$. The scattered light was collected at a fixed angle of $90^{\circ}$. The autocorrelation function was processed by applying the Stokes-Einstein equation and the particle size distributions were calculated from the translational diffusion coefficient.

To evaluate the secondary structure content and to score the overall folding of Pdx1, Pdx2, and the Pdx complex, CD spectroscopy investigations were performed. CD spectra of the samples were recorded at $20{ }^{\circ} \mathrm{C}$ by utilizing a J-815 CD spectrometer (Jasco, UK). The mean molar ellipticity ( $\mathrm{deg} \mathrm{cm}^{2} \mathrm{dmol}^{-1}$ ) was plotted against the wavelength applying the Spectra Manager ${ }^{\mathrm{TM}}$ software, and the secondary structure contents were determined with the Spectra Manager ${ }^{\mathrm{TM}}$ software [23].

\subsection{Solution Small Angle X-Ray Scattering (SAXS)}

Pdx1, Pdx2, and the Pdx complex were analyzed by SAXS. Data were collected at the EMBL beamline P12 (PETRA III, DESY, Hamburg, Germany). For complex formation, pure Pdx1 and Pdx2 were mixed in a 1:1 ratio and incubated overnight. Prior to SAXS data collection, the complex was purified by a Superose-6-increase column (10/300, GE Healthcare). Pdx1 and the Pdx complex were investigated at concentrations of 1,2 , and $5 \mathrm{mg} \mathrm{mL}^{-1}$ by applying an automated robotic sample changer and a Dectris 2D photon-counting detector (PILATUS-6M) with $3.1 \mathrm{~m}$ sample to detector distance. Considering the oligomerization tendency of $\mathrm{Pdx2}$, online size-exclusion chromatography was applied prior to SAXS data collection (SEC-SAXS) by utilizing $7 \mathrm{mg} \mathrm{mL}^{-1} \mathrm{Pdx} 2$ and a Superdex-75-increase column (5/150, GE Healthcare), which was pre-equilibrated with a buffer of $20 \mathrm{mM}$ Tris- $\mathrm{HCl}$ (pH 8.0), $200 \mathrm{mM} \mathrm{NaCl}, 1 \mathrm{mM}$ EDTA, $2 \mathrm{mM}$ DTT, and 3\% glycerol. The obtained eluted sample solution was directly subjected to $\mathrm{X}$-ray scattering data collection.

Scattering data for all three samples were collected, integrated, and averaged by applying the SasTool software (EMBL, Hamburg, Germany) [43]. Guinier analysis and radius of gyration $\left(\mathrm{R}_{\mathrm{G}}\right)$ values were calculated using PRIMUS [29]. The pair distribution functions $P(r)$ and forward scattering intensities I(0) were processed with GNOM [44] and PRIMUS [29]. Obtained data were compared with the values provided by SAXSMoW [35]. Kratky plots $\left(\mathrm{I}_{(\mathrm{S})} \mathrm{S}^{2}\right.$ versus $\left.\mathrm{S}\right)$ and Porod-Debye plots $\left(\mathrm{I}_{(\mathrm{S})} \mathrm{S}^{4}\right.$ versus $S$ ) were generated, as described previously [45], and molecular mass (MM) data were obtained from SAXSMoW [35]. Low-resolution ab initio models were generated using the programs DAMMIN and GASBOR, considering the symmetry option P1 for Pdx2 and symmetry P6 for Pdx1 and the Pdx complex [46,47]. Rigid body models were constructed applying the program SASREF [48] considering 
the same symmetry options as those used for the dummy models. Theoretical scattering curves of homologous structures deposited at the protein data bank were calculated, compared, and fitted to the experimental data using CRYSOL [30].

\subsection{Electron Microscopy (EM) Analysis}

For EM investigations, sample solutions with concentrations between 25 and $30 \mu \mathrm{g} \mathrm{mL}{ }^{-1}$ of Pdx1, $\mathrm{Pdx} 1$ in complex with Pdx2 monomers, and Pdx1 in complex with Pdx2 oligomers in $20 \mathrm{mM}$ Tris $\mathrm{pH}$ 8.0 containing $200 \mathrm{mM} \mathrm{NaCl}$ and $5 \mathrm{mM} \mathrm{MTG}$ (monothioglycerol) were stained with $2 \%$ uranyl acetate. Specimens were prepared for EM investigations using a conventional negative staining procedure [49]. Sample solution drops of $4 \mu \mathrm{L}$ were adsorbed to a glow-discharged, carbon-coated, copper grid, washed with two drops of deionized water, and stained with two drops of freshly prepared $2 \%$ uranyl acetate solution. Micrographs were recorded with a Talos L120C (Thermo Fisher Scientific-FEI) electron microscope at $120 \mathrm{kV}$ accelerating voltage and a LaB6 source filament with a CETA camera. Images were taken using a magnification of $71,000 \times$ to $92,000 \times$.

\section{Conclusions}

We presented a detailed analysis of the $P$. vivax Pdx proteins, $\mathrm{Pdx} 1$ and $\mathrm{Pdx} 2$ in solution, that form the pyridoxal phosphate (PLP) biosynthesis enzyme complex and provided structural details about the P. vivax Pdx1 dodecamer, the oligomerization behavior of $\mathrm{Pdx} 2$, and the dynamics of $\mathrm{Pdx}$ complex formation. Our investigations applying complementary bioanalytical techniques showed that purified P.vivax Pdx1 formed a stable dodecamer in solution and that purified $\mathrm{Pdx} 2$ is present as a monomer but forms different oligomers in solution over time. Time-resolved DLS experiments revealed the dynamics of Pdx complex formation and completion in hours by monitoring a solution of $\mathrm{Pd} x 1$ dodecamer and adding Pdx2. In contrast to Pdx1, the PLP synthase domain of the pyridoxal phosphate (PLP) biosynthesis complex, purified Pdx2 could be detected in different oligomeric states; interestingly, however, the complex exhibited a distinct tendency to specifically interact with Pdx1, resulting in a hetero-oligomeric $\mathrm{Pdx}$ complex irrespective of the monomeric or oligomeric state of $\mathrm{Pdx} 2$ in solution. These results highlight that the Pdx1 dodecamer is the essential and stable subunit in the $\mathrm{Pdx}$ complex acting as an anchor, and $\mathrm{Pdx} 2$ can bind to the $\mathrm{Pdx} 1$ dodecamer upon availability.

Supplementary Materials: Supplementary materials can be found at http://www.mdpi.com/1422-0067/21/17/ 5971/s1.

Author Contributions: Conceptualization, N.U., C.B. and C.W.; methodology, N.U., H.A. and C.N.M.; software, N.U. and S.F.; validation, C.B., C.W. and S.F.; investigation, N.U., H.A. and C.N.M.; resources, C.B. and C.W.; writing—original draft preparation, N.U. and H.A.; writing—review and editing, N.U., S.F. and C.N.M.; supervision, C.B. and C.W.; project administration, C.B. and C.W.; funding acquisition, N.U., H.A., C.B. and C.W. All authors read and agreed to the published version of the manuscript.

Funding: The authors acknowledge financial support by the Cluster of Excellence "Advanced Imaging of Matter" of the Deutsche Forschungsgemeinschaft (DFG)-EXC 2056, project ID 390715994, by BMBF via project 05K16GUA and a support grant from HEC, Pakistan, under the project "Faculty Development Program BZU Multan (100 PhD Scholarships) (Prime Minister's Directive)". Further, the authors are grateful to the Fundação de Amparo à Pesquisa do Estado de São Paulo (FAPESP, grants 2017/03966-4 and 2015/26722-8).

Acknowledgments: The authors acknowledge the support of beamline scientists of the EMBL beamline P12 (PETRA III, DESY) and Cryo-EM staff scientists of CSSB (Hamburg) for supporting the EM experiments.

Conflicts of Interest: The authors declare no conflict of interest.

\section{Abbreviations}

AUC Analytical ultra-centrifugation

BZU Bahauddin zakariya university

CD Circular dichroism

CSSB left for structural systems biology

DESY Deutsches electronen-synchrotron 


$\begin{array}{ll}\text { DLS } & \text { Dynamic light scattering } \\ \text { Dmax } & \text { Maximum diameter } \\ \text { EM } & \text { Electron microscopy } \\ \text { EMBL } & \text { European molecular biology laboratory } \\ \text { HEC } & \text { Higher education commission } \\ \text { I(s) } & \text { Scattering intensity } \\ \text { MM } & \text { Molecular masses } \\ \text { MTG } & \text { Monothioglycerol } \\ \text { Ni-NTA } & \text { Nickel nitrilotriacetic acid } \\ \text { NTA } & \text { Nanoparticle tracking analysis } \\ \text { PAGE } & \text { Polyacrylamide gel electrophoresis } \\ \text { PDB } & \text { Protein data bank } \\ \text { Pdx } & \text { Pyridoxal } \\ \text { Pr } & \text { Distance distribution function } \\ \text { PLP } & \text { Pyridoxal phosphate } \\ \text { Pv } & \text { Plasmodium vivax } \\ \text { Pf } & \text { Plasmodium falciparum } \\ \mathrm{R}_{\mathrm{G}} & \text { Radius of gyration } \\ \mathrm{R}_{\mathrm{H}} & \text { Hydrodynamic radius } \\ \text { RMS } & \text { Root mean square deviation } \\ \text { SAXS } & \text { Small angle x-ray scattering } \\ \text { SDS } & \text { Sodium dodecyl sulphate } \\ \text { SEC } & \text { Size-exclusion chromatography } \\ \rho & \text { Shape factor } \\ & \end{array}$

\section{References}

1. World Health Organization. World Malaria Report 2019; World Health Organization: Geneva, Switzerland, 2019.

2. Amadi, A.; Ezeonu, I.; Akoma, O. Antimalarial Drug Resistance: An Existential Burden for the Developing World. Microbiol. Res. J. Int. 2019, 1-16. [CrossRef]

3. World Health Organization. Global Technical Strategy for Malaria 2016-2030; World Health Organization: Geneva, Switzerland, 2015.

4. Patel, T.S.; Bhatt, J.D.; Dixit, R.B.; Chudasama, C.J.; Patel, B.D.; Dixit, B.C. Design and synthesis of leucine-linked quinazoline-4 $(3 \mathrm{H})$-one-sulphonamide molecules distorting malarial reductase activity in the folate pathway. Arch. Pharm. 2019, 352, 1900099. [CrossRef] [PubMed]

5. Baird, J.K.; Price, R.N.; Zimmerman, P.A.; Gething, P.W.; Battle, K.E. Growing evidence of Plasmodium vivax across malaria-endemic Africa. PLOS Negl. Trop. Dis. 2019. [CrossRef]

6. World Health Organization. Control and Elimination of Plasmodium Vivax Malaria: A Technical Brief; World Health Organization: Geneva, Switzerland, 2015.

7. Müller, I.B.; Knöckel, J.; Groves, M.R.; Jordanova, R.; Ealick, S.E.; Walter, R.D.; Wrenger, C. The assembly of the plasmodial PLP synthase complex follows a defined course. PLoS ONE 2008, 3, e1815. [CrossRef]

8. Liang, J.; Han, Q.; Tan, Y.; Ding, H.; Li, J. Current advances on structure-function relationships of pyridoxal 5'-phosphate-dependent enzymes. Front. Mol. Biosci. 2019, 6. [CrossRef]

9. Wrenger, C.; Eschbach, M.-L.; Müller, I.B.; Warnecke, D.; Walter, R.D. Analysis of the vitamin B6 biosynthesis pathway in the human malaria parasite Plasmodium falciparum. J. Biol. Chem. 2005, 280, 5242-5248. [CrossRef]

10. Knöckel, J.; Jordanova, R.; Müller, I.B.; Wrenger, C.; Groves, M.R. Mobility of the conserved glycine 155 is required for formation of the active plasmodial Pdx1 dodecamer. Biochim. Biophys. Acta Gen. Subj. 2009, 1790, 347-350. [CrossRef]

11. Rodrigues, M.J.; Windeisen, V.; Zhang, Y.; Guédez, G.; Weber, S.; Strohmeier, M.; Hanes, J.W.; Royant, A.; Evans, G.; Sinning, I. Lysine relay mechanism coordinates intermediate transfer in vitamin B6 biosynthesis. Nat. Chem. Biol. 2017, 13, 290-294. [CrossRef] 
12. Flicker, K.; Neuwirth, M.; Strohmeier, M.; Kappes, B.; Tews, I.; Macheroux, P. Structural and thermodynamic insights into the assembly of the heteromeric pyridoxal phosphate synthase from Plasmodium falciparum. J. Mol. Biol. 2007, 374, 732-748. [CrossRef]

13. Neuwirth, M.; Flicker, K.; Strohmeier, M.; Tews, I.; Macheroux, P. Thermodynamic Characterization of the Protein- Protein Interaction in the Heteromeric Bacillus subtilis Pyridoxalphosphate Synthase. Biochemistry 2007, 46, 5131-5139. [CrossRef]

14. Zhu, J.; Burgner, J.W.; Harms, E.; Belitsky, B.R.; Smith, J.L. A new arrangement of ( $\beta / \alpha) 8$ barrels in the synthase subunit of PLP synthase. J. Biol. Chem. 2005, 280, 27914-27923. [CrossRef] [PubMed]

15. Strohmeier, M.; Raschle, T.; Mazurkiewicz, J.; Rippe, K.; Sinning, I.; Fitzpatrick, T.B.; Tews, I. Structure of a bacterial pyridoxal 5'-phosphate synthase complex. Proc. Natl. Acad. Sci. USA 2006, 103, 19284-19289. [CrossRef] [PubMed]

16. Kim, S.; Kim, K.-J. Crystal structure of Mycobacterium tuberculosis Rv2606c: A pyridoxal biosynthesis lyase. Biochem. Biophys. Res. Commun. 2013, 435, 255-259. [CrossRef] [PubMed]

17. Neuwirth, M.; Strohmeier, M.; Windeisen, V.; Wallner, S.; Deller, S.; Rippe, K.; Sinning, I.; Macheroux, P.; Tews, I. X-ray crystal structure of Saccharomyces cerevisiae Pdx1 provides insights into the oligomeric nature of PLP synthases. FEBS Lett. 2009, 583, 2179-2186. [CrossRef]

18. Bauer, J.A.; Bennett, E.M.; Begley, T.P.; Ealick, S.E. Three-dimensional structure of YaaE from Bacillus subtilis, a glutaminase implicated in pyridoxal-5'-phosphate biosynthesis. J. Biol. Chem. 2004, 279, $2704-2711$. [CrossRef]

19. Gengenbacher, M.; Fitzpatrick, T.B.; Raschle, T.; Flicker, K.; Sinning, I.; Müller, S.; Macheroux, P.; Tews, I.; Kappes, B. Vitamin B6 Biosynthesis by the Malaria Parasite Plasmodium falciparum BIOCHEMICAL AND STRUCTURAL INSIGHTS. J. Biol. Chem. 2006, 281, 3633-3641. [CrossRef]

20. Zein, F.; Zhang, Y.; Kang, Y.-N.; Burns, K.; Begley, T.P.; Ealick, S.E. Structural insights into the mechanism of the PLP synthase holoenzyme from Thermotoga maritima. Biochemistry 2006, 45, 14609-14620. [CrossRef]

21. Smith, A.M.; Brown, W.C.; Harms, E.; Smith, J.L. Crystal structures capture three states in the catalytic cycle of a pyridoxal phosphate (PLP) synthase. J. Biol. Chem. 2015, 290, 5226-5239. [CrossRef]

22. Guédez, G.; Hipp, K.; Windeisen, V.; Derrer, B.; Gengenbacher, M.; Böttcher, B.; Sinning, I.; Kappes, B.; Tews, I. Assembly of the eukaryotic PLP-synthase complex from Plasmodium and activation of the Pdx1 enzyme. Structure 2012, 20, 172-184. [CrossRef]

23. Yang, J.T.; Wu, C.-S.C.; Martinez, H.M. Calculation of protein conformation from circular dichroism. In Methods in Enzymology; Elsevier: Amsterdam, The Netherlands, 1986; Volume 130, pp. 208-269.

24. Dierks, K.; Meyer, A.; Einspahr, H.; Betzel, C. Dynamic light scattering in protein crystallization droplets: Adaptations for analysis and optimization of crystallization processes. Cryst. Growth Des. 2008, 8, 1628-1634. [CrossRef]

25. Falke, S.; Betzel, C. Dynamic Light Scattering (DLS). In Radiation in Bioanalysis; Springer: Berlin/Heidelberg, Germany, 2019; pp. 173-193.

26. Putnam, C.D.; Hammel, M.; Hura, G.L.; Tainer, J.A. X-ray solution scattering (SAXS) combined with crystallography and computation: Defining accurate macromolecular structures, conformations and assemblies in solution. Q. Rev. Biophys. 2007, 40, 191-285. [CrossRef] [PubMed]

27. Burchard, W.; Schmidt, M.; Stockmayer, W. Information on polydispersity and branching from combined quasi-elastic and intergrated scattering. Macromolecules 1980, 13, 1265-1272. [CrossRef]

28. Mukherjee, A.; Hackley, V.A. Separation and characterization of cellulose nanocrystals by multi-detector asymmetrical-flow field-flow fractionation. Analyst 2018, 143, 731-740. [CrossRef] [PubMed]

29. Konarev, P.V.; Volkov, V.V.; Sokolova, A.V.; Koch, M.H.; Svergun, D.I. PRIMUS: A Windows PC-based system for small-angle scattering data analysis. J. Appl. Crystallogr. 2003, 36, 1277-1282. [CrossRef]

30. Svergun, D.; Barberato, C.; Koch, M.H. CRYSOL-A program to evaluate X-ray solution scattering of biological macromolecules from atomic coordinates. J. Appl. Crystallogr. 1995, 28, 768-773. [CrossRef]

31. Waterhouse, A.; Bertoni, M.; Bienert, S.; Studer, G.; Tauriello, G.; Gumienny, R.; Heer, F.T.; de Beer, T.A.P.; Rempfer, C.; Bordoli, L. SWISS-MODEL: Homology modelling of protein structures and complexes. Nucleic. Acids. Res. 2018, 46, W296-W303. [CrossRef]

32. Roy, A.; Kucukural, A.; Zhang, Y. I-TASSER: A unified platform for automated protein structure and function prediction. Nat. Protoc. 2010, 5, 725-738. [CrossRef] 
33. Altschul, S.F.; Gish, W.; Miller, W.; Myers, E.W.; Lipman, D.J. Basic local alignment search tool. J. Mol. Biol. 1990, 215, 403-410. [CrossRef]

34. DeLano, W.L. The PyMOL Molecular Graphics System. Available online: http://www.pymol.org (accessed on 1 July 2020).

35. Fischer, H.; Oliveira Neto, M.d.; Napolitano, H.; Polikarpov, I.; Craievich, A.F. Determination of the molecular weight of proteins in solution from a single small-angle $\mathrm{X}$-ray scattering measurement on a relative scale. J. Appl. Crystallogr. 2010, 43, 101-109. [CrossRef]

36. World Health Organization. Guidelines for the Treatment of Malaria; World Health Organization: Geneva, Switzerland, 2015.

37. Divo, A.A.; Geary, T.G.; Davis, N.L.; Jensen, J.B. Nutritional requirements of Plasmodium falciparum in culture. I. Exogenously supplied dialyzable components necessary for continuous growth. J. Protozool. 1985, 32, 59-64. [CrossRef]

38. Derrer, B.; Windeisen, V.; Rodríguez, G.G.; Seidler, J.; Gengenbacher, M.; Lehmann, W.D.; Rippe, K.; Sinning, I.; Tews, I.; Kappes, B. Defining the structural requirements for ribose 5-phosphate-binding and intersubunit cross-talk of the malarial pyridoxal 5-phosphate synthase. FEBS Lett. 2010, 584, 4169-4174. [CrossRef] [PubMed]

39. Ventura, S. Sequence determinants of protein aggregation: Tools to increase protein solubility. Microb. Cell Factories 2005, 4, 11. [CrossRef] [PubMed]

40. Fernandez-Escamilla, A.-M.; Rousseau, F.; Schymkowitz, J.; Serrano, L. Prediction of sequence-dependent and mutational effects on the aggregation of peptides and proteins. Nat. Biotechnol. 2004, 22, 1302-1306. [CrossRef] [PubMed]

41. Raschle, T.; Amrhein, N.; Fitzpatrick, T.B. On the two components of pyridoxal $5^{\prime}$-phosphate synthase from Bacillus subtilis. J. Biol. Chem. 2005, 280, 32291-32300. [CrossRef]

42. Robert, X.; Gouet, P. Deciphering key features in protein structures with the new ENDscript server. Nucleic Acids Res. 2014, 42, W320-W324. [CrossRef]

43. Franke, D.; Petoukhov, M.; Konarev, P.; Panjkovich, A.; Tuukkanen, A.; Mertens, H.; Kikhney, A.; Hajizadeh, N.; Franklin, J.; Jeffries, C. ATSAS 2.8: A comprehensive data analysis suite for small-angle scattering from macromolecular solutions. J. Appl. Cryst. 2017, 50, 1212-1225. [CrossRef]

44. Svergun, D. Determination of the regularization parameter in indirect-transform methods using perceptual criteria. J. Appl. Cryst. 1992, 25, 495-503. [CrossRef]

45. Rambo, R.P.; Tainer, J.A. Characterizing flexible and intrinsically unstructured biological macromolecules by SAS using the Porod-Debye law. Biopolymers 2011, 95, 559-571. [CrossRef]

46. Svergun, D.I. Restoring low resolution structure of biological macromolecules from solution scattering using simulated annealing. Biophys. J. 1999, 76, 2879-2886. [CrossRef]

47. Svergun, D.I.; Petoukhov, M.V.; Koch, M.H. Determination of domain structure of proteins from X-ray solution scattering. Biophys. J. 2001, 80, 2946-2953. [CrossRef]

48. Petoukhov, M.V.; Svergun, D.I. Global rigid body modeling of macromolecular complexes against small-angle scattering data. Biophys. J. 2005, 89, 1237-1250. [CrossRef] [PubMed]

49. Ohi, M.; Li, Y.; Cheng, Y.; Walz, T. Negative staining and image classification-Powerful tools in modern electron microscopy. Biol. Proced. Online 2004, 6, 23-34. [CrossRef] [PubMed]

(C) 2020 by the authors. Licensee MDPI, Basel, Switzerland. This article is an open access article distributed under the terms and conditions of the Creative Commons Attribution (CC BY) license (http://creativecommons.org/licenses/by/4.0/). 